\title{
Corrigendum: Altered Gray Matter Volume in Patients With Type 1 Diabetes Mellitus
}

\section{OPEN ACCESS}

Approved by: Frontiers Editorial Office, Frontiers Media SA, Switzerland

*Correspondence: Haojun Shi doc_shi@163.com Qing Zhu xhzhuqing@126.com Jing Wang

jjwinflower@126.com

Specialty section:

This article was submitted to Neuroendocrine Science,

a section of the journal

Frontiers in Endocrinology

Received: 23 July 2020

Accepted: 24 July 2020

Published: 28 August 2020

Citation:

Liu J, Fan W, Jia Y, Su X, Wu W, Long X,

Sun X, Liu J, Sun W, Zhang T, Gong Q, Shi H, Zhu Q and Wang J (2020) Corrigendum: Altered Gray Matter Volume in Patients With Type 1 Diabetes Mellitus. Front. Endocrinol. 11:604. doi: 10.3389/fendo.2020.00604

\begin{abstract}
Jia Liu ${ }^{1,2}$, Wenliang Fan ${ }^{1,2}$, Yuxi Jia ${ }^{1,2}$, Xiaoyun Su ${ }^{1,2}$, Wenjun Wu ${ }^{1,2}$, Xi Long $^{1,2}$, Xin Sun ${ }^{1,2}$, Jie Liu ${ }^{1,2}$, Wengang Sun ${ }^{1,2}$, Tianjing Zhang ${ }^{3}$, Qiyong Gong ${ }^{4}$, Haojun Shi ${ }^{1,2 *}$, Qing Zhu ${ }^{5 *}$ and Jing Wang ${ }^{1,2 *}$

${ }^{1}$ Department of Radiology, Union Hospital, Tongji Medical College, Huazhong University of Science and Technology, Wuhan, China, ${ }^{2}$ Hubei Province Key Laboratory of Molecular Imaging, Wuhan, China, ${ }^{3}$ Philips Healthcare, Guangzhou, China, ${ }^{4}$ Department of Radiology, Huaxi MR Research Center, West China Hospital of Sichuan University, Chengdu, China, ${ }^{5}$ Department of Neurology, Union Hospital, Tongji Medical College, Huazhong University of Science and Technology, Wuhan, China
\end{abstract}

Keywords: gray matter, type 1 diabetes mellitus, seed-based d mapping, meta-analysis, voxel-based morphometry

\section{A Corrigendum on}

Altered Gray Matter Volume in Patients With Type 1 Diabetes Mellitus

by Liu, J., Fan, W., Jia, Y., Su, X., Wu, W., Long, X., et al. (2020). Front. Endocrinol. 11:45. doi: 10.3389/fendo.2020.00045

In the published article, there was an error in affiliations 1 and 5. Instead of "Department of Radiology, Tongji Medical College, Union Hospital, Huazhong University of Science and Technology, Wuhan, China," it should be "Department of Radiology, Union Hospital, Tongji Medical College, Huazhong University of Science and Technology, Wuhan, China."

The error in affiliation 5 should be instead of "Department of Neurology, Tongji Medical College, Union Hospital, Huazhong University of Science and Technology, Wuhan, China," it should be "Department of Neurology, Union Hospital, Tongji Medical College, Huazhong University of Science and Technology, Wuhan, China."

The authors apologize for these errors and state that this does not change the scientific conclusions of the article in any way. The original article has been updated.

Copyright $\odot 2020$ Liu, Fan, Jia, Su, Wu, Long, Sun, Liu, Sun, Zhang, Gong, Shi, Zhu and Wang. This is an open-access article distributed under the terms of the Creative Commons Attribution License (CC BY). The use, distribution or reproduction in other forums is permitted, provided the original author(s) and the copyright owner(s) are credited and that the original publication in this journal is cited, in accordance with accepted academic practice. No use, distribution or reproduction is permitted which does not comply with these terms. 